\title{
The Metapattern of General Evolutionary Dynamics and the Three Dynamical Realms of Big History
}

Tyler Volk

Professor of Biology and Environmental Studies, New York University

Correspondence I Tyler Volk, tyler.volk@nyu.edu

Citation I Volk, Tyler. 2020. “The Metapattern of General Evolutionary Dynamics and the Three Dynamical Realms of Big History." Journal of Big History 4 (3): 31-53.

DOI I https://doi.org/10.22339.jbh.v4i3.4320

\section{K E Y W O R D S}

Big History

combogenesis

grand sequence

dynamical realms

general evolutionary dynamics

biological evolution

cultural evolution

metapatterns

\begin{abstract}
A B S T R A C T
The goal of this paper is to formalize better the division of big history into three main stages (phases, eras). In my own work they are "dynamical realms," 1. physical laws, 2. biological evolution, and 3. cultural evolution. I show a deep similarity in two mighty transitions; first, from dynamical realm 1 to 2 , and then from 2 to 3 . The common "metapattern" in these transitions is that of generalized evolutionary dynamics, which in both cases opened up vast new arenas of possibility space. I first present relevant conclusions from my book, Quarks to Culture. A "grand sequence" of twelve fundamental levels was forged through a repeated cycle of "combogenesis" spanning the dynamical realms as families of levels. Next, I provide examples of other scholars who have similarly weighed in on a three-fold arc; notably Christian, Spier, Chaisson, Rolston, Salk, and Voros (following Jansch). Like me, all have nominally recognized similarities between biological and cultural evolution as important in the dynamics of realms two and three. Generally, these scholars have not placed primary emphasis on general evolutionary dynamics as a multiply-instantiated process. The PVS metapattern for evolution (propagation, variation, and selection) is well established as overarching across many patterns in biology, following life's origin. In culture the operation of general evolutionary dynamics is, I suggest, dual-tier, consisting of cognitive PVS of individuals coupled to social PVS of groups. The emergence of realm-forming PVS-dynamics twice (biology, culture) created radically new ways to explore and stabilize patterns in expansive fields of diverse types within the respective dynamics. Thus, we can recognize a fundamentally similar reason (i.e., two emergent forms of evolutionary dynamics) for why so many scholars have correctly, in my opinion, discerned a threefold arc of big history. Important as well in the flow of progress from quarks to culture were two only slightly less major instantiations of PVS-dynamics (though both crucial): an era of chemical evolution within the realm of physical laws, which led into the realm of biological evolution, and also the evolution of the animal cognitive learning PVS of trial, error, and success, which was essential to the path into cultural evolution. In concluding remarks, I note several outstanding issues: alternative proposals for five orders or four dimensions (i.e., divisions more than three in the arc of big history); the use of the word "evolution," and three matrices (cosmosphere, biosphere, civisphere) that contain and are constituted by the varieties of patterns within the corresponding dynamical realms.
\end{abstract}

\section{Introduction: Patterns and Metapatterns}

Patterns are fundamental to big history. A focus on patterns allows us to formulate a unified field of study, linking things as disparate as protons, stars, oceans, amoebas, trees, ancient cities of Mesopotamia, and democracies. Toward this end, David Christian's (2011, 505) words are spot on: "Of all the patterns that occur at many different scales, the most fundamental is the existence of pattern itself."

We patternologists are concerned not only with patterns of things. Things have relations (affordances, capabilities). Thus, relations are an important type of pattern for big history. Jack 
Pearce $(2018,1)$ writes: "the visible, tangible Universe is the set of continuing, progressive correlations between interacting elements, forming systems of relationships." Lowell Gustafson (2017) emphasizes "sustained patterned relations," at various scales, from atoms in molecules to people in polities.

In the terms of Fred Spier (2015), things are "building blocks," relations are "connections," and over time the universe has produced "sequences." Sequences should be considered as another general type of pattern in big history; for example, David Christian's (2018) series of thresholds. With sequences, we explicitly bring in the flow of time, and thus changes in patterns and the creation of new patterns.

In Metapatterns (Volk 1995), I discussed a "metapattern" of "sequences of stages." In this metapattern found across scales, stages of relative continuity are ratcheted one to the next by "breaks," or changes of state. Examples are the stages of insect metamorphosis or stages in Zen's ox-herding pictures of enlightenment.

I usually use the term metapattern to refer to a pattern that exists in both biology and culture, a sort of super-convergence (Volk and Bloom 2007). Considering super-convergence makes more concrete Gregory Bateson's term "metapattern," which he somewhat enigmatically defines as a "patterns of patterns" (1979 preface). My own interest has been to seek general patterns of form-and-function that have been mostly independently discovered by pattern-making processes in biology and culture, including the mind.

We could say that certain generalities for big history already mentioned, such as sequences, building blocks, connections, thresholds, and even pattern itself are all metapatterns. So would be other big history principles, such as Goldilocks conditions or gradients. In this paper, the main metapattern to which I draw attention will be that of general evolutionary dynamics; namely, propagation, variation, and selection (PVS, or PVS dynamics). In addition, the concept of a dynamical realm is also a metapattern.

Therefore, considering the (meta)pattern of sequences of stages, we will see that a number of scholars have developed models for big history that contain, at the very largest scale of time, a threefold arc. Despite its prevalence in the big history literature (and I include those too early to have called themselves big historians, for example, Jonas Salk; see below), the threefold sequence has not really been spotlighted in big history work. At least I have not seen it referred to as the pattern that deserves focused analysis, nor analyzed in enough depth as a pattern that itself should cause us, I submit, to go "wow."

Therefore, what I will do here first is offer a précis of conclusions relevant to this paper from my book Quarks to Culture (Volk 2017). Then I will note evidence from others for a three-stage, largest scale sequence from cosmos to culture. Crucial to this paper is what I will call general evolutionary dynamics (or, simply evolutionary dynamics), to be spelled out as a particular kind of dynamics for how things of various classes explore possibilities and turn into new patterns.

In essence, to the extent we accept an overall threefold arc of big history, the rules of the games played by systems changed in major ways twice. Both transitions created new types of evolutionary dynamics that were different and yet that also shared a deep similarity by possessing component processes of propagation, variation, and selection (PVS). To round out the picture, we will also develop concepts about chemical evolution and animal cognition as PVS dynamics, both important precursor forms of dynamics leading into the respective next realms of biology and culture.

I will conclude with comments on the use of the word "evolution," about sequences other than the threefold one proposed here, and about other future challenges, all outstanding issues that deserve further consideration.

\section{Combogenesis, Twelve Fundamental Levels, and Three Dynamical Realms}

In Quarks to Culture (Volk 2017, here $Q_{2} C$ ), I derived twelve fundamental levels. These twelve progressed from the first level, which includes quarks and the other fundamental particles, to the twelfth, initiated by the ancient geopolitical states. Today's world that is going 'planetary' is possibly a level 13 . 


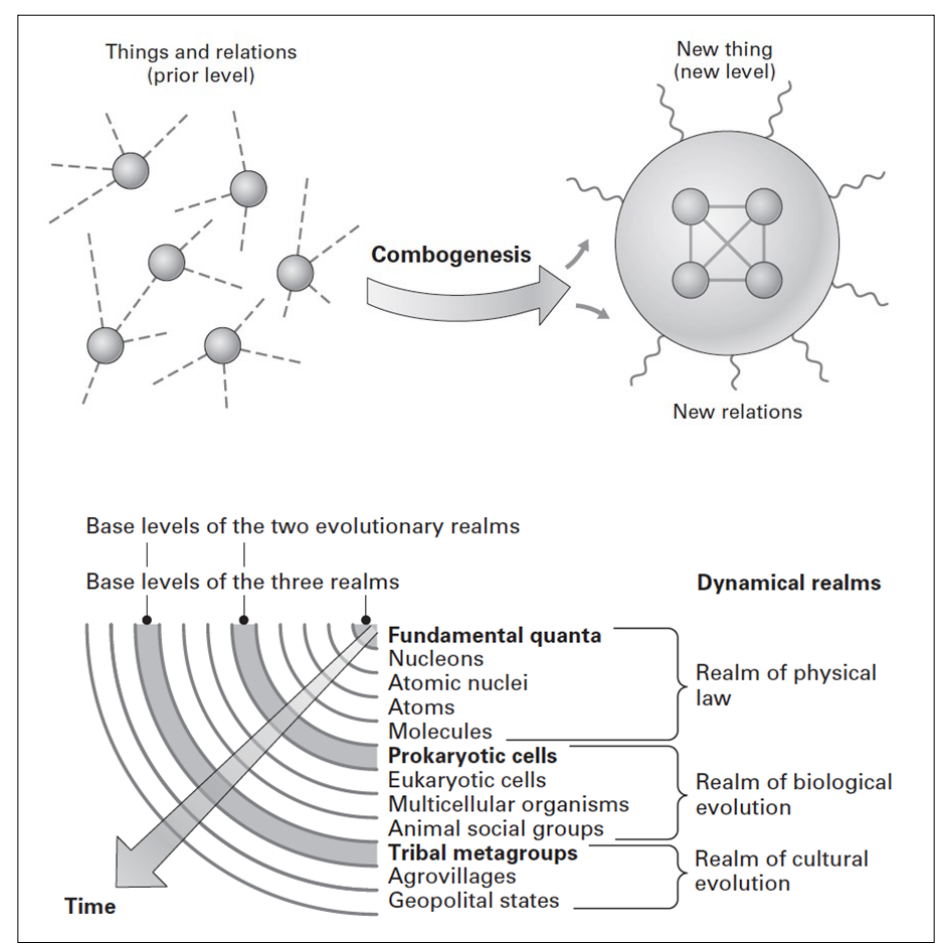

Figure 1. Upper: The logic of combogenesis. The circles on the left are the things (systems, entities) of a prior level. The dotted lines represent the relations they have with each other and with all things in their environment. The single larger circle on the right is the new type of thing (system, entity) on the new level, which results from combination and integration, combogenesis; the wavy lines radiating from the circle represent the new kinds of relations it has and is capable of having. Lower: The three dynamical realms, with particular spans across groups of levels. The base levels of the three dynamical realms are shaded in the concentric circles and are labeled in bold type in the list of levels. Note that two base levels (prokaryotic cells and tribal metagroups) initiate the specific evolutionary realms that are the focus of this paper.

I derived these levels by applying a particular logic. In a nutshell, the logic, called combogenesis, starts with the simplest things (building blocks and their relations) established by physics. Then one works along time, noting first origins of types that form a sequence of nested build-ups by combination and integration. I stay within the path of progression from small to large, toward the human body (as a special something we care about; which technically is a member of level eight, multicellularity). Then the logic continues from the body to human social systems (as things we are within and that could exist only after we as bodies existed).

The twelve levels derived from this logic are 1. Fundamental particles; 2. Nucleons; 3. Atomic nuclei; 4. Atoms; 5. Molecules; 6. Prokaryotic cells; 7. Eukaryotic cells; 8. Multicellular organisms; 9. Animal social groups; 10. Human tribal metagroups; 11. Agrovillages; and 12. Geopolitical states. See Figure 1 for this resulting "grand sequence."

In $Q_{2} C$ I provided pointers to what was new at each level, and what were the key new attributes that enabled the formation of each next, subsequent level, considering the specific dynamics of combination and integration as things grew in scale and fundamental type from each level to the next. Furthermore, the early members of any given level could not have been achieved directly from the things from types two or more levels down. Space does not allow me to review the specific evidence and reasoning for each level of combogenesis in this special, unabashedly human focused grand sequence. Some additional discussion will come in later when relevant to specific purposes here.

From the fundamental levels, three groupings - three families of levels - virtually pop out. I call these three families the "dynamical realms." Before getting into the dynamical realms, let us recognize as data for this paper the fact that others have noted three largest scale groupings as well, which I will review in the section after next. Such convergence in the parsing of big history would seem to indicate an important pattern to think about. First, I will define and illustrate the concept of a dynamical realm.

\section{Dynamical Realms and Core Dynamics across Levels in Each}

According to my terminology, the three dynamical realms are (1) physical laws, (2) biological evolution, and (3) cultural evolution. Their relationship to the twelve fundamental levels can be seen in Figure 1.

I have described a dynamical realm as follows $\left(Q_{2} C_{151}\right)$ :

A dynamical realm is a series of levels that share special, governing operations-that is, dynamics. These dynamics are core processes of the workings of things and relations common across the levels that constitute the realm. The implication is that spans of levels form categories larger than the individual 
levels themselves, promptly suggesting the existence of certain large-scale themes of the kind we seek. Thus, a dynamical realm is a kind of world or zone or space of behaviors. We see its core workings continue when general aspects of explanatory logic repeat across levels for specific events of combogenesis. The core processes under focus involve the methods of stabilizing things shared across various levels.

Thus, the things in the dynamical realm of physical laws - such as nucleons, atoms, molecules, planets, stars, galaxies, minerals, raindrops-all share and are basically explainable by fundamental forces of physics (and various balances, increases and decreases, concatenations, and subtle modifications of the forces). Specifically, using the model of the grand sequence of combogenesis, in this realm we have the fundamental levels of 1 . the standard model, 2. nucleons, 3. atomic nuclei, 4. atoms, and 5 . molecules prior to the origin of life. In a recent paper, Voros (2019) skillfully shows connections between the micro-scale build ups of these levels and macro-scale things such as planets, stars and galaxies. Such connections are an important topic but not the subject of this paper. (See, however, the concluding remarks concerning my proposal for three "super-spheres" of context that came into existence at the same time as the base levels of the three dynamical realms.)

To continue, the things in the dynamical realm of biological evolution-such as living cells, plants, animals, DNA, ribosomes, chlorophyll, ecosystems, biosphere, ant colonies, chimp communities-all share and, for explanation, need the pattern-finding process of biological evolution. Specifically, using the model of the grand sequence of combogenesis, in this realm we have levels 6 . the prokaryotic cell and the origin of life, 7. the eukaryotic cell, 8. multicellular organisms, and 9. animal social groups. Importantly, there is a difference between types of things in this realm that can be directly subject to life-death comparative dynamics of biological evolution (such as the free-living prokaryotic cell or an animal) and those larger types that contain living things (i.e., communities, ecosystems, biosphere) but are not directly subject to evolution, even though they need those directly evolvable things in our explanations for their structure and formation. (All still use physics, of course-the dynamics of one realm do not stop when the next realm starts.)

Finally, the things in the dynamical realm of cultural evolution-such as conscious people, hunter-gatherer groups, cultivated wheat, samurai swords, the alphabet, medieval city walls, Plato's Republic, a Fender Stratocaster, and modern democracies, election districts, and billionairesall share and, for explanation, need the patternfinding process of cultural evolution. Specifically, using the model of the grand sequence of combogenesis, in this realm we have 10. the human tribal metagroup and the origin of cultural evolution, 11. agrovillages, and 12. geopolitical states. In my proposed model, we are still in level 12 of the geopolitical state, though it has changed greatly from the ancient states to modern nations (similar to the way that worms becoming whales was a path of change within the level of animal multicellularity $\left(Q_{2} C 128,140\right)$. Like the examples in biology above, things in this realm are subject to the dynamics of cultural evolution to a greater or lesser degree, even though all require cultural evolution as parts of the explanations we give them for understanding.

This model implies that particular levels are 'base levels' that began respective dynamical realms. Again, see Figure 1. Base levels initiated those specific new core dynamics that are so crucial to understanding steps of combogenesis to subsequent levels that continued those core dynamics. The base levels are (realm 1, level 1) the fundamental starter-stuff of physics, (realm 2, level 6) prokaryotic cells, and (realm 3, level 10) human cultural metagroups.

If the proposal in this paper is correct (or gains support from a few readers), we can expect something fundamentally new at the base levels of the dynamical realms of biological and then cultural evolution. The remainder of this paper looks in more detail at these evolutionary dynamical realms 2 and 3, the transitions into them from their respective prior realms, and the importance of the multiple instantiations of the metapattern of general evolutionary dynamics, in both form- 
ing new evolutionary realms and in lead-ups into them.

\section{A Threefold Big History Structure Noted by Other Scholars}

A number of scholars have proposed a similar threefold, largest scale sequence from the Big Bang to us. Examples come from David Christian,
Fred Spier, Eric Chaisson, Holmes Rolston III, Jonas Salk, and Joseph Voros. Table 1 summarizes references and terms.

Table 1 provides evidence of seven scholars who note a threefold structure to big history. In some cases, they cite each other, indicating agreement or borrowing. In my opinion, for the

\begin{tabular}{|c|c|c|c|c|}
\hline Scholar & Overall Term & $\begin{array}{c}\text { Term for Dynamical } \\
\text { Realm } 1\end{array}$ & $\begin{array}{c}\text { Term for Dynamical } \\
\text { Realm } 2 \\
\end{array}$ & $\begin{array}{c}\text { Term for Dynamical } \\
\text { Realm } 3 \\
\end{array}$ \\
\hline Christian (1) & $\begin{array}{c}\text { Big History } \\
\text { Main parts of recent book }\end{array}$ & Cosmos & Biosphere & Us \\
\hline Spier (2) & $\begin{array}{c}\text { Big History } \\
\text { Major types of complexity }\end{array}$ & $\begin{array}{c}\text { Physical inanimate } \\
\text { nature }\end{array}$ & Life & Culture \\
\hline Chaisson (3) & $\begin{array}{c}\text { Cosmic Evolution } \\
\text { Phases }\end{array}$ & Physical Evolution & Biological Evolution & Cultural Evolution \\
\hline Rolston (4) & $\begin{array}{c}\text { Three Big Bangs } \\
\text { Universal evolution's } \\
\text { three phases }\end{array}$ & Matter-Energy & Life & Mind \\
\hline Salk (5) & $\begin{array}{l}\text { Evolution of } \\
\text { Complexity }\end{array}$ & $\begin{array}{l}\text { Pre-biological } \\
\text { Evolution }\end{array}$ & Biological Evolution & $\begin{array}{l}\text { Metabiological } \\
\text { Evolution }\end{array}$ \\
\hline Voros (6) & $\begin{array}{c}\text { Phases of Cosmic } \\
\text { Evolution's } \\
\text { Grand Sequence }\end{array}$ & $\begin{array}{c}\text { Cosmologic Evolution } \\
\text { (Physical) }\end{array}$ & $\begin{array}{c}\text { Sociobiological } \\
\text { Evolution } \\
\text { (biological) }\end{array}$ & $\begin{array}{c}\text { Sociocultural Evolution } \\
\text { (cultural) }\end{array}$ \\
\hline Volk (7) & Dynamical Realms & Physical Laws & Biological Evolution & Cultural Evolution \\
\hline
\end{tabular}

Table 1: Multiple descriptions of a threefold great sequence

All terms are direct quotes from the sources. Terms may vary within a source, but this table shows what I judge to be each source's most representative terms for the great threefold sequence.

(1) David Christian (2018). Terms are titles of his book's main parts that elaborate on his eight thresholds, in one or more chapters per threshold within these parts.

(2) Fred Spier (2015). His focus, well known to the big history (BH) community, is on the relationship between energy and complexification within the sequence from physics to culture.

(3) Eric Chaisson $(2014,2015)$. Also well known to the BH community, Chaisson focuses on a universal metric of "energy flux density" and its increase over "cosmic evolution." Spier (2015, 58-59) identifies "a great many complications" but does conclude, "Chaisson's analysis seems fair enough as a first-order approach."

(4) Holmes Rolston III (2010). This book, Three Big Bangs, is not commonly cited in the $\mathrm{BH}$ literature but deserves applause and is directly relevant here.

(5) Jonas Salk (1985). This remarkable paper is worth attention by the BH community. It appeared in a volume honoring Salk's friend Jacob Bronowski. Salk's term "metabiological" is basically what many of us would call culture. See also Salk's book, Anatomy of Reality, 1983.

(6) Joseph Voros (2019, following Jantsch 1980). Voros does a service to the $\mathrm{BH}$ community to bring up the prescient work of Eric Jantsch. Do not be perplexed by the term "sociobiological evolution;" it is basically what several others call biological evolution. Also, Voros has a worthwhile discussion about the use of the term "cosmic evolution."

(7) Volk (2017). 
most part, we should view these as independent results, showing a convergence of ideas.

Despite differences in terminology, the three divisions closely flow vertically in the table. We will ignore tricky and debatable issues about how the authors describe the beginnings of each of the three divisions. For one thing, not everyone is concerned with exact beginnings. Second, such beginnings are very much still being worked out by relevant discoveries. Indeed, the births of the three realms hold some of the most demanding questions for science $\left(Q_{2} C_{1}\right.$ 188-190).

Without doubt many more examples of the threefold structure could be brought in from other scholars with notable exceptions, to be discussed toward the end. For now, let us take this convergence as meaning something. But what?

We should at least consider an answer relying on an 'observer effect': the divisions might not really be there. They might be human creations. Humans have a tendency to see things in time as having stages. We reify gradients drawn from continua by drawing lines and then putting names on stages. As ancestral humans sought God, perhaps today's big history scholars seek major stages. In my judgment this would constitute a lazy answer that would not do justice to the findings of science (though in all cases of scholarship, we do have to be cautious about the human projection of patterns).

Let us consider then that this table points to what might be an important pattern for big history. Yet, I have not seen much targeted discussion specific to this tripartite division.

To emphasize, there frankly has not been enough inquiry into this. Yes, there has been excitement about thresholds, complexity, energy flux density, and other proposed principles, as metapatterns cutting across the threefold sequence but not much about the pattern of the three sub-arcs of the entire arc. In hopes of adding some "wow," let us inquire more deeply into how this threefold pattern came about.

\section{Possibility Space and the Exploration, For- mation, and Stabilization of Patterns; or, Games that Patterns Play}

The convergence implies that a number of scholars see two giant shifts in the deep nature of the universe's myriad things and relations: first, from physics-chemistry into biology, and second, from biology into humans and culture. As suggested in my analysis to come (though I look forward to being corrected), we could be making more of a point that the emergence of new, multilevel-enabling dynamics happened twice using different instantiations of the same core dynamics. In simplest terms, I think everyone in the table would agree that at some point in time on Earth, life came on the scene. That changed a lot of things in a big way! Then, at some subsequent point, humans arrived. Once again, that changed a lot in a big way!

There is a conceptual issue here. It involves the phrase, "in a big way." In my model of a grand sequence, innovations in things and their relations come into existence with each and every one of the twelve new levels of combogenesis (or, to take another model, with each one of Christian's eight thresholds). Such innovations of levels or thresholds were all big. However, at certain, special levels (or thresholds) the innovations were so momentous that they opened the doors to entire families of subsequent levels (or thresholds). How do we determine what created the truly momentous versus the merely big in the degree of innovation?

Holmes Rolston III (2010, 33-37) has wrestled with this conundrum, and thus the question of scales of innovation, through the concept of "possibility space." Rolston points out that in some sense, everything now existing was born in the possibility space set up at the Big Bang. Given the starter-set stuff of basic particles and rules of our universe, then jet planes and democraciesas possibilities-were 'there' at the moment of the physical Big Bang. Yet we also know that jet planes and democracies-as materialized patterns-would not have come into being unless living cells (and many other things, such as atoms, stars, etc.) emerged in sequence in the cosmic cavalcade.

Although the Big Bang opened up the total set of all possibilities we have seen manifested, something is lacking from that too simple a viewpoint. Rolston's answer (if I may interpret it as such) is his concept of "three big bangs." Following the 
first big bang, what made the next two big bangs that launched life and culture so momentous was that those two origins each opened up huge new possibility spaces. The new ways of exploring patterns opened up by the starts of biology and culture allowed the creation of new fundamental levels (or thresholds) within those new two major ways of operating.

This concept of types of possibility space and how new things are achieved out of possibilities is directly related to Spier's (2015, 48-54) three basic forms of complexity (physical, biological, cultural). To have a trio of main types of complexity follow one upon the other in a sequence implies that twice new types of complexity came about from previous types. These new types were not just different, but extraordinarily impactful in that they allowed further complexifications within those basic types. The starts of these types of complexity created new dynamical realms (in my language), with families of levels within each. One might think of nestings of scales (or degrees) of innovation.

We also might think of the grand situation in the following way. Two times the rules of the processes that created patterns, relations, and sequences (smaller than and thus within the three realms of the grand sequence) changed in ontology-expanding ways. If physics and chemistry are simple, analogous, say, to the game of tic-tac-toe, then the rules of the biological games, once invented, might be more like checkers. Continuing, then culture might be more like chess (or fill in with your favorite complex game here, such as Go, or Risk). Rules of the three games allow different methods of probing possibilities, bounded, of course, to certain ranges of actualization.

I support the view that the new games are new ways of exploring possibility space. This paper's claim is that the pattern of the threefold arc of big history was made because twice new, major types of general evolutionary dynamics started. First came the dynamical realm of biological evolution from the non-evolutionary realm of physical laws. Then came the dynamical realm of cultural evolution from that of biological evolution.

I will next go into biological evolution, and then to culture, showing the relationship be- tween evolutionary dynamics and possibility space, in terms of exploring and stabilizing patterns. The issue of scale will again come in.

\section{The Dynamical Realm of Biological Evolu- tion; Things with Imports of Nutrients and Exports of Wastes}

I think it is no exaggeration to say that a preponderance of big history scholars see the origin of life as a crucial start to a new era of patterns on Earth. That is certainly true of those cited in table 1. For example, with the big bang of life "the rules of the game change ... and the future is like no previous past" (Rolston 2010, 82).

A more complete list of thinkers with similar views would be expansive. We would include, for example, Schrödinger's insights (1944, What is Life?) into new types of entropy fluxes and information storage at the origin of life, as well as Greg Henriques and colleagues (2019) with their new "dimension of existence" that began with life.

Similarly, it is no exaggeration to say that most would specifically highlight the overarching process of biological evolution as a game changer that ratcheted up the complexity with a new playground of patterns, like progressing from the humdrum of tic-tac-toe to the upscale challenge of checkers. As John Stewart (2019) says, "given sufficient numbers of generations, complex adaptations could be discovered by this trial-and-error searching of possibility space." (Perhaps Dobzhansky's famous quote is already reverberating in your mind: "Nothing in biology makes sense except in the light of evolution.")

Yet there are many who point not only to evolution but also to the entity called the living, prokaryotic cell as key at life's origin, and specifically drawing from a list of descriptions that typically could include items such as DNA, negentropy, autopoiesis, boundary membrane, reproduction, information, CHNOPS, ribosomes, metabolism, reproduction, and more.

The importance of many items in such lists as key at life's start is not in dispute here. We can see issues of scale, particularly in two main scales of innovations. Yes, the prokaryotic cell was not only an innovation with a list of factors. On a 
much larger scale, the cell also started a new dynamical realm with the pattern-exploring game of biological evolution. Thus, we should try and integrate the local, internal dynamics of the living cell upward into the larger, population-scale dynamics of evolution, the process by which prokaryotic cells could change in time, resulting in stupendously radiating and sometimes even merging lineages of life.

To bridge these two scales of cell and evolution, I concur with the logic of Fred Spier (2015, 146): "The complexity of life is fundamentally different from more simple forms of complexity, because it actively harvests matter and energy from outside .... Because resources are finite, at a certain point competition inevitably set in." Spier goes on to note that at this transition point, basically the process of evolution set in.

I would like to add to Spier's note of the cell's need for "harvest," which involves a transfer from a cell's outside to its inside. Let us consider as well the complementary process of waste ejection-from inside to outside. Important for generating biological evolution is the fact that both directions of fluxes will degrade the environment around the cell $\left(Q_{2} C\right.$ 75-77). With respect to a cell's ability to continue living, its imports reduce the surrounding nutrient concentrations. A cell's exports increase the surrounding waste concentrations, which are presumably toxic or in some way detrimental (after all, the cell ejected the wastes as necessary for its metabolism).

Given this pair of negative effects as a double whammy, then, returning to Spier's terms, competition set in. Furthermore, because cells vary, selection follows. (Variation is a large topic, not to be discussed here because I assume it is not controversial in general, but at base the complexities of a cell's internal metabolism and the process of replication lead to inadvertent variations.)

We now have the connection between the two scales of dynamics. As a consequence from what the living cell does at its local scale of living dynamics, the larger scale of biological evolution follows. Of course, these scales intertwine and coproduce each other. Yet what a cell does itself is more fundamental. Evolutionary dynamics are an inadvertent consequence.
Biological evolution is a "blind watchmaker," in Richard Dawkins' famous phrase. It is blind, yet powerful. Though not a directly selected feature of living cells, evolution at life's origin constituted the core operations that established a new dynamical realm of pattern-shaping. Biological evolutionary dynamics were able to engender a series of subsequent levels (figure 1) because things made at those levels continued to possess the core operations that create evolutionary dynamics.

To elaborate, key to the core operations that caused evolution to cascade into the future of life are that key pair of fluxes: imports of nutrients and exports of wastes. After the prokaryotic cell (level 6), the eukaryotic cell (level 7) also imports and exports. So does the multicellular organism (level 8). Level 9 of the animal social group is trickier. If we disregard the controversy of "group selection," the animals (which contain eukaryotic cells, which contain mitochondria) in a social group have collective imports and exports summed from the number of individuals. Furthermore. individual animals are subject to evolution, within the context of a group. (To be clear, similar-species groups are what I consider to be level 9.) The animal social group is generally not subject to direct life-death dynamics as a whole thing. There is a close tie between the individual animal in certain groups that have social learning and the larger-scale, behavioral organization of a group itself, a topic not to be expanded here. I assume it is not controversial to this readership that evolution can work on individual animals to facilitate their behavioral adaptations for life in complex groups.

Thus, evolutionary dynamics continued on all these levels that followed the origin of the prokaryotic cell. Evolutionary dynamics followed as a consequence of what living things do to live. Again, in this view, the imports and exports are the essential, fundamentally new relations of the things with the new form of complexity at the base level of life. These relations continue up the levels of the grand sequence.

\section{PVS Dynamics as a Metapattern}

Next let us generalize evolution as a metapattern. There is P: propagation, a necessity of living 
things. There is V: variation, noted earlier. There is S: selection. All together, this is: PVS, or PVS dynamics. This trio of sub-processes together make the 'recipe' for evolution. As a scale independent metapattern, PVS dynamics need not be confined to biology.

The generalized evolutionary process has been called by various names: an algorithm (Dennett 1995), a recipe (Wilson 2007), a formula (Buskes 2013). Its sub-processes are commonly three in number, like my PVS. These go by slightly different names, which will not be debated here. Though I prefer the term propagation over equivalent alternatives for the $\mathrm{P}$ subprocess, others use reproduction, replication, or inheritance. The term variation is almost universal. So is the term selection. As one more example of how terms can vary, instead of selection, Spier (2015, following Chaisson) prefers "non-random elimination," and psychologist Donald Campbell (1960) uses "selective retention."

Two brief asides suggest one can tease out these three sub-processes and how they work to produce evolution in Darwin's famous final paragraph of The Origin of Species. Also, the PVS as a recursive system is, of course, not a standard cycle like the phases of the moon; it is more like a braid whose length is time and in which all strands are interwoven in the ongoing generation of patterns. See Figure 2.

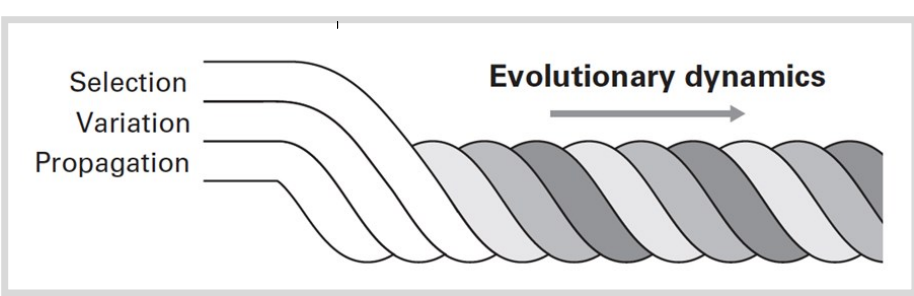

Figure 2. Evolutionary dynamics (in the general sense not limited to biology) consists of three component processes, depicted here as the interwoven strands of a braid of sub-processes: propagation, variation, and selection.

The philosopher Chris Buskes (2013), in his paper, "Darwinism Extended: A Survey of How the Idea of Cultural Evolution Evolved," makes cogent points about what he calls "Darwin's formula" and how it is applicable to both biological and cultural evolution. The "three elements" of this logic constitute an overall dynamical process that is substrate neutral. Daniel Dennett also notes the importance of substrate neutrality in his general evolutionary "algorithm." Whether formula, algorithm, or recipe, the application of PVS dynamics over and over again produces "cumulative selection" (Buskes 2013), and thus cumulative change, from bacteria to brontosauruses in the case of biology, and from hand axes to hand sanitizers in that of culture.

The application of the term evolution to culture requires more discussion to support my aim to promote the PVS metapattern as a key principle in big history. For example, what about the $\mathrm{P}$ sub-process? Propagation as a general subprocess clearly needs to be more expansive than the self-propagation of life forms, such as in biological reproduction. A discussion on how to expand $\mathrm{P}$ to culture will come. First, to show a clear application of evolution as a metapattern, let us turn to what many have called "chemical evolution," which led 'up' and into biological evolution.

\section{Chemical Evolution Preceded Biological Evolution}

We can now make a first concrete application of the PVS metapattern to a region of pattern formation that is outside biology. For this we go first not directly forward in time's grand sequence to culture, but backward, to chemistry.

Many scholars have talked about "chemical evolution" as a transition to the origin of life. Chemical evolution refers to the ability of molecules to make autocatalytic loops that can complexify by PVS dynamics operating upon those loops as variable wholes. What gives the loops (or nested systems of loops, see Kauffman, 2019) the ability to evolve follows as a consequence of their complexity, with the inherent potential for small variants to arise and differentially propagate.

One theorist whose formulation I have found particularly useful is the theoretical and organic chemist Addy Pross. In papers and books (e.g., Pross 2012; Pross and Pascal 2017), he and colleagues distinguish "thermodynamic stability" from "dynamic kinetic stability" (DKS). Briefly, the more complex chemical DKS systems (the autocatalytic loops in the preceding paragraph) can 
exhibit evolution because they require imports of fresh substrate molecules as feed stocks in their semi-closed reactions. They also require the ejection as exports of dead-end 'waste' molecules made within their internal reaction loops. Thus, DKS chemical systems are import-export things. They were possible within what I call the level of molecules within the dynamical realm of physical laws.

In his newest book, A World Beyond Physics, Stuart Kauffman (2019) shows how these pre-life, biochemical DKS systems would inherently propagate as chemical 'things,' how they would inherently vary by blebbing off sets of sub-reactions and accepting blebs from others, and thus how these DKS systems could indeed evolve by cumulative change. The DKS things explore possibility space and manifest new forms from what Kauffman calls "the adjacent possible."

Because of their export-import lifestyles, such DKS systems would compete and thus improve (dare we use that word?) in their possession of internal functionalities that support their 'lives,' as they progress in time from simple chemistry toward the complex prokaryotic cell at the base level of life.

Yes, so much about getting from complex chemistry to the base level of life is still damn mysterious. Nonetheless this scenario has growing experimental evidence and theoretical support, involving entities that Eörs Szathmáry (2015) calls "protocells."

If we accept the basic outline, then the relevance for the proposed PVS metapattern for big history is that chemical PVS dynamics preceded and led into full on biological PVS dynamics. Chemical evolution transited into biological evolution, which from our distance in time, was a major leap.

\section{Should the era of chemical evolution be con- sidered a separate dynamical realm?}

This is an interesting, open question, worthy of discussion. I do not expect it to be answerable in an absolute way. Even so, I raise it now and will return to it again later in the paper.

On one hand, if we use the start of a new form of PVS as the definitive mark for a new dynamical realm, then it might seem that the answer is "yes." This viewpoint should be considered.

On the other hand, I am inclined to a soft, albeit noncommittal, "no." For one, in the chemical wilderness of nature today we apparently do not confirm protocells or autocatalytic loops clearly on the path toward life. If this absence of evidence continues, it would appear that the postulated types of primordial chemical loops have disappeared, perhaps as they long ago chemically evolved and merged into the operations of the cell of the Last Universal Common Ancestor at the origin of life and biological evolution. However, back-pedaling a bit, if we have not found the wild protocells yet, we should be open to what might be out there awaiting discovery (perhaps in deep sea vents).

Another, related reason for my soft "no" is that the two forms of PVS-dynamics in the scenario sketched-chemical PVS leading to biological PVS-are in some sense so continuous in time that they might seem to be not independent inventions of the PVS metapattern. Both chemical and biological evolution involve selection upon variants of propagating chemical patterns of import-export systems (for a moment, biology considered as complex chemistry). Thus, the first transitioned-how smoothly, it is unknowninto the other.

I think the case is much stronger when we come to cultural evolution, for a new evolutionary dynamical realm that arises from an innovative instantiation of PVS dynamics. To that we turn next.

\section{Cultural Evolution Is a Form of Evolutionary Dynamics, Dual-tier with Both Individual and Social PVS Dynamics}

We find a real breakthrough in the manifestation of the PVS metapattern when we consider cultural evolution. The innovation is substantial enough to call the result a new dynamical realm. I will show that the overall PVS dynamics of cultural evolution is a system of both mental and social operations that, therefore, couples two tiers of specific (because to some degree separable) PVS. 
The awareness of culture as evolutionarymore than metaphorically so-goes deep in the big history literature. As shown in Table 1, many scholars explicitly call culture a form of evolution. Even those who do not use the word in the table use the term at times in their writings. For example, Christian (2018, 335) cites Alex Mesoudi, one of the leaders in cultural evolution theory. Spier sees teacups and large buildings created by "adaptive radiations" within the "artificial complexity" component of his third major type of general complexity, that of culture (Spier 2015, 253).

I suggest that cultural evolution-and specifically as a new realization of the PVS metapattern-should become (even) more prominent in the big history literature. One advantage for upping the focus is that this entangled power trio of $\mathrm{P}-\mathrm{V}-\mathrm{S}$ can be studied by unraveling it into its separate players. There might be rich potential for integrative scholarship here: How is cultural possibility space explored by variants at all scales of culture from material objects to conceptual systems such as religion or science? How are cultural patterns selected, by degrees-inadvertently, individually, and collectively across multiple scales of groups? What various mechanisms that change over time propagate these patterns? What about the conscious mind? Recent work is spotlighting the high fidelity, complex copying abilities of humans and also their specific innovation of teaching (Laland 2017; Buskes 2019; Tomasello 2019).

Using evidence from the anthropological literature, I have proposed a transition of combogenesis from animal social groups (level 9) to human tribal metagroups (level 10; $Q_{2} C$ Ch. 13). This is key: tribal metagroups (i.e., beginning with hunter-gatherers in extended social organizations) were not just a larger group of primates but consisted of local 'post-primate' groups networked into a larger cultural sea of groups. The metagroup is thus a new fundamental level of combination and integration. Indeed, this new level was so significant that it launched not just a new level but a unique dynamical realm because its 'things' possessed cultural evolutionary dynamics.
The new, extended and extendable social organizations depended on language and material things such as tools, shelters, and symbolic material artifacts. Language and material culture served as mutually-reinforcing, interstitial bindings among people, integrating local, smaller groups into landscape-spanning, fuzzily-bounded socio-cultural 'things.'

A larger-scale social organization was one necessary condition for the emergence of cumulative cultural evolution (Tomasello 2019, 19). The larger 'thing' maintained itself broad enough to be selfpropagating. In this new level, people in a local group could stay connected in the larger metagroup to others who might be living on the other side of a mountain or kilometers down the river.

That culture was evolutionary-in the sense that at some point it had PVS-dynamics-is supported in detail by the many scholars cited earlier (for a good overview, see Buskes 2013). Truly, cultural PVS of some sort cannot be controversial. Look around at restaurants as flowers beckoning to potential pollinators from pedestrian throngs along Bleecker Street in the Big Apple (not at the moment, in April 2020, but hopefully again soon). Cultural PVS dynamics are often brought up with regard to information, but I like to point to examples that more directly drive the point home, such as restaurants or cars.

Many-Buskes and Tomasello have been cited but there are many-use the term "cumulative" culture (the apt metaphor of a cultural "ratchet" is also popular). My only slightly new point along these lines has been to place culture in sequence as a new dynamical realm, worth formally defining in the entire grand sequence as realm number three, and which began in a particularly innovative fundamental level of the tribal metagroup during the recursive process of combogenesis. The key to the innovative quality of that level was the explosive upsurge of pattern-making powers from the new form of PVS.

More specifically, I claim that the special, human form of cultural dynamics can be usefully studied and positioned as dual-tier PVS $\left(Q_{2} C\right.$ 177180). The two tiers are as follows:

Tier one: There is PVS in the minds of individuals (more about this in a bit). Mental PVS has 
been recently noted in this journal (Stewart 2019; Voros 2019).

Tier-two: There is social PVS, in the group decision-making systems that began at least by the time of the extended societies of huntergatherers, the exemplars for the original metagroup origination. This social PVS is largely operational within the daily groups. Relevant to his emphasis on "collective learning," Christian (2018: 178) points to contacts and thus vital networks among neighboring groups. There is even evidence that Neanderthals had occasional multiband gatherings (Hayden 2012) as crucial to their culture. Indeed, I envision the invention of the active use of the word "we" as some sort of threshold. See, for example, the focal importance of "we-intentionality" of John Searle (Plotkin 2003) or the unique human capacity during a child's development to progress from joint intentionality to collective intentionality (Tomasello 2019).

Putting these two tiers together-and they likely coevolved in early cultural evolutionproduced cultural systems that enabled a 'Rolstonian' third 'big bang.' The dual-tier cultural systems opened up vast new types of possibility spaces and allowed newly generated cultural patterns to undergo expansions and progressions.

\section{Learning as PVS Dynamics in Cultural Evolution}

Learning has much been emphasized in big history literature as key to the human realm, and for many good reasons. What I would like to suggest here is we might try and merge big history's emphasis on learning to an equal emphasis on dual-tier PVS dynamics in cultural evolution. We should explore what learning and dual-tier PVS might have to do with each other. I think it is a very close relationship.

Spier $(2015,141)$ sees the correspondence between biological evolution and human history close enough to call "both . . . driven by learning processes." One can therefore surmise that learning contains PVS dynamics. I mean that as a strong statement. I would guess that, ultimately, learning is likely the larger set of phenomena. If so, it would be interesting to distinguish the PVS parts of learning (of various kinds) from the non-
PVS parts. Perhaps, as Christian's system of collective learning (2018) includes both decisionmaking (which clearly is PVS) and modeling, this would make learning larger than the PVS portion of learning. One's models improve as well, and thus are progressively created from a PVS process that likely has both cognitive and social components. If an anatomization of cultural evolution could be figured out, a general analysis of the role of learning in big history might benefit.

For example, scholars noted above cite teaching as a major innovation that helped forge humanity in a step from a more basic social learning like that possessed by fission-fusion animal social groups. If the scholars are right, then teaching would be one large innovation of the $P$ (propagation) function of cultural evolution's overall PVS. Laland (2017) notes how culture requires, above all, the primary ability to employ cultural mechanisms for propagation. Teaching is also a new form of selection in its complex, personal evaluations.

\section{Once Invented, the Continuation of Cultural Evolutionary Dynamics in the Grand Sequence}

From a perspective on big history, we can see a metapattern repeated: a major new form of PVS dynamics came in with culture, and culture was linked to the form.

Similar to the way that the PVS of biology continued into the biological levels of the grand sequence, the dual-tier, cognitive-social PVS of culture continued into subsequent cultural levels. Once started, cognitive PVS systems of internal "testing" of ideas and scenarios and decisionmaking were always there and linked to evolving, social forms of decision-making, scenariobuilding, and attempts at persuasion and influence, to note just a few forms of social PVS available.

Once started, the overall, combined, dual-tier PVS of culture continued into innovations and complexifications (inexorable from our retrospective perspective?). A new fundamental level within the dynamical realm of culture came about when the metagroup structure of level 10 incorporated plants and animals into the human cultural system, leading to level 11 of agrovillages. 
Then came level 12 of geopolitical states, in which the main innovation was the discovery of how to do socio-cultural political combogenesis with takeover and incorporation by various power games, enabled by the innovation of the extendable bureaucracy $\left(\mathrm{Q}_{2} \mathrm{C}, 137 \mathrm{ff}\right.$.). All these advancements required minds with cognitive PVS dynamics and social PVS operations for making group decisions and incorporating changes that emerged as trials involving at various scales language and material culture.

\section{From Animal Cognition to Human Dual-tier PVS and a Full-on Cultural Dynamical Realm}

Given that biological evolution was in place with its inadvertent PVS, then PVS as a metapattern could potentially be found by biological evolution, if the pattern forming properties of a PVS system was advantageous. In other words, the pattern or process of PVS could be discovered and established within an organism as an adaptation. One well known example is the adaptive immune system possessed by most vertebrates (Cziko 1995).

How did the transition to the major innovation of cultural evolution occur? My proposal will be limited to showing the involvement of a biological type of PVS dynamics as an adaptation prior to the PVS dynamics of cultural evolution $\left(Q_{2} C, 176 f f.\right)$. The specific claim is that animal cognitive PVS led to the more complex PVS of human mind, a key partner of the dual-tier PVSsystem that also included an explicit social-PVS for full-on cultural evolution.

Here is where learning as a PVS system comes in again. Learning is a tricky concept and, in some interpretations, could even be applied to behavioral changes of a bacterium (LeDoux 2019). Pertinent to our seeking for PVS as an evolved adaptation directly relevant to human evolution, the neuroscientist Joseph LeDoux also emphasizes how crucial was the step of animal evolution that enabled more complex trial-and-error learning as an adaptation (specifically, Thorndikian learning). In animal evolution, this type of learning was a cognitive advance from one-shot, simple conditioned learning, which can happen, for example, in threat-response conditioning.
In the way LeDoux sees this transition, simple trial and error behavior was supplemented by mental modeling in some mammals. Then primates developed forms of cognitive deliberation to simulate mentally their trial-and-error experiments and avoid some of the risks of harm or even death always present in actual tests of behavior. Another leap occurred in humans with language and especially syntax and hierarchical reasoning (personal communication). The question of consciousness, or its gradual evolution, is of course open and controversial.

Trial-and-error animal learning, therefore, is a PVS system that shapes relatively sophisticated behavior from successful experience (and from failure, which can induce behavioral extinction or a switch in behavior). The idea described is that the animal brain of sufficient complexity has some sort of PVS cognitive 'metabolism.' Let us call it animal PVS-learning. These cognitive operations are useful to an animal's life.

Once evolved, the basic operation of animal cognitive PVS could be further sculpted and complexified by biological evolution. As described, the PVS capacity of animal learning, as a useful adaptation, could progress in a ratchet into more and more powerful mental PVS-systems to advanced, multi-layered human PVS in the brainmind. Somewhere back in time, in our ancestral lineage, culture emerged from abilities that ever more advanced PVS-learning enabled.

The importance of an innovation in animal learning is consistent with Henriques and colleagues' (2019) proposal that animal cognition is a landmark new "dimension," because it involved a "behavioral selection" feedback loop that "builds mental complexity" from "variation, selection, and retention." Their proposal for this new dimension will be revisited toward the end of this paper.

It seems reasonable that this cognitive PVS of animals (again, of a certain complexity) grew in potential and operational power during the genesis of cultural humans along with social abilities, as noted above. These changes in the mental and social capabilities would have at first both been linked closely to biological evolution but then more and more were running on their own, cou- 
pled in special cultural evolutionary dynamics (Boyd and Richerson 1985). Specifically, the human mind became capable of imaginative prospection. We even make individual, whole-life choices or selection, from ancients deciding to go on a vision quest, to moderns making career plans.

John Stewart nails it: $(2019,142)$ cognitively, an "evolutionary process was internalized within the minds of humans." Stewart also notes the oftencited words of Karl Popper (1972), as this capability "permits our hypotheses to die in our stead." Similarly, Rolston discusses the importance of "ideational variation" $(2010,98)$ in the cultural big bang.

It is important to state that the individual's mental PVS was most likely not an inadvertent byproduct. In this way, the animal cognitive PVS is thus unlike the overall process of biological evolution's PVS, which at its start was an inadvertent consequence of the metabolic import and export fluxes of the cell. The difference is that animal cognitive PVS evolved within the animal as a biological adaptation with advantages for the animal.

In my view, to repeat, in the progress to humans from animal cognitive PVS (say, of the level of chimps or bonobos), the cognitive PVS cooperated with and was coupled to changes that led to human social structures, in particular the metagroup systems that enabled culture. Thus, we need to bring in the social and some significant innovation of social PVS dynamics. This is consistent with the views of numerous theorists, such as Tomasello (2019).

How do the human tiers of cognitive and social PVS connect? Details are beyond the scope of this paper and my mind. Specifics are easily seen to be truly entangled and complex. For example, one's own thought processes are subjects of discussion by others, even groups of others, who then influence the individual via training with encouragement and its opposite. We would have to consider here all the complexity that social networks have as control systems for propagating culture as individuals enter and leave through births and deaths. The cultural anthropologist Christopher Boehm $(2012,354)$ has described an ancient era in which bands had "fierce egalitarianism" (based on hunter-gatherer data), which they maintained by group discussions and decisions, for example, about how to handle a problem individual.

Today humans possess both internal complex decision-making processes that range in their degree of complexity. For example, decision-making modalities divide into fast and slow mental systems (Kahneman 2011). More generally, we weigh options and juggle possibilities in an inner cognitive possibility space, filled with yeses and nos, and these decisions are connected to intricate demands and nuances of support and rejection from others, and those 'others' can be groups in both real and even imagined social spaces (Luhmann 2012).

This is all generally consistent with Christian's discussion of the importance of collective learning (2018) in the emergence of culture. My specific emphasis here is on the PVS metapattern, and the repeated manifestation of it as a principle in big history, for the dynamical realms of biology and culture, because of that metapattern's power in exploring possibility space and bringing in the real from the unmanifest. Discoveries and syntheses are gradually filling in the specifics of the still greatly mysterious transition from biology to culture (Tomasello 2019; Laland 2017; many others), so my broad-brush answer is therefore limited to a perspective that uses the PVS metapattern as a principle to propose large-grain structures in that remarkable emergence.

The transition would have been fuzzy in time, of course. I do not see how it would be pinned down precisely, but we can see its brush strokes when far enough away, sometime from the emergence of hominids to the Upper Paleolithic. My personal review of the literature $\left(Q_{2} C\right.$ 115-117) would place the transition by or in the Middle Paleolithic (also, Mesolithic, or Old Stone Age, a term used for the same period in African paleoarcheology).

Parallels from Using the Metapattern of PVS Dynamics as a Logical Principle That Is Invented Multiple Times and When Modified Radically Enough Creates Particularly Consequential Thresholds 
We now can synthesize the main points above to show several parallels between the emergence of the two great evolutionary realms of biology and culture.

The singular major parallel has been noted well enough: these two realms deserve to be called "evolutionary" because they each initiated a new form of PVS that facilitated multiple subsequent fundamental levels of combogenesis. The respective families of levels in biology and culture all share realm-unique, core operations of their respective forms of PVS. I will assume that this point has been made adequately so that a reader at least knows what I am putting forward for evaluation.

A second parallel occurred in the ways that the base levels of the two evolutionary realms, with their realm-forming PVS innovations, were formed from earlier, transitional PVS dynamics born in the advanced levels of the previous realm. This parallel took place in the way that the transi- tions into the realms of biology and then culture used transfers and then further changes of a prior PVS-system that continued 'up' from the terminal levels of the respective previous dynamical realms. (To be clear, by "terminal" I mean only patterns within a realm that had innovations that we can see led directly into a next realm. The terminal levels were thus launch pads, not dead-end terminations).

I will elaborate on the two parts of this second parallel:

First, the emergence of life: Within the realm of physical laws, molecules (level 5 , see Figure 1) were able to complexify into autocatalytic loops of chemical reactions with chemical PVS dynamics. This type complex chemistry with dynamickinetic stability (DKS) was capable of exploring molecule-space in an era of chemical evolution, which led into the profound shift to the origin of life (level 6) and the start of the dynamical realm of biological evolution. See Figure 3.

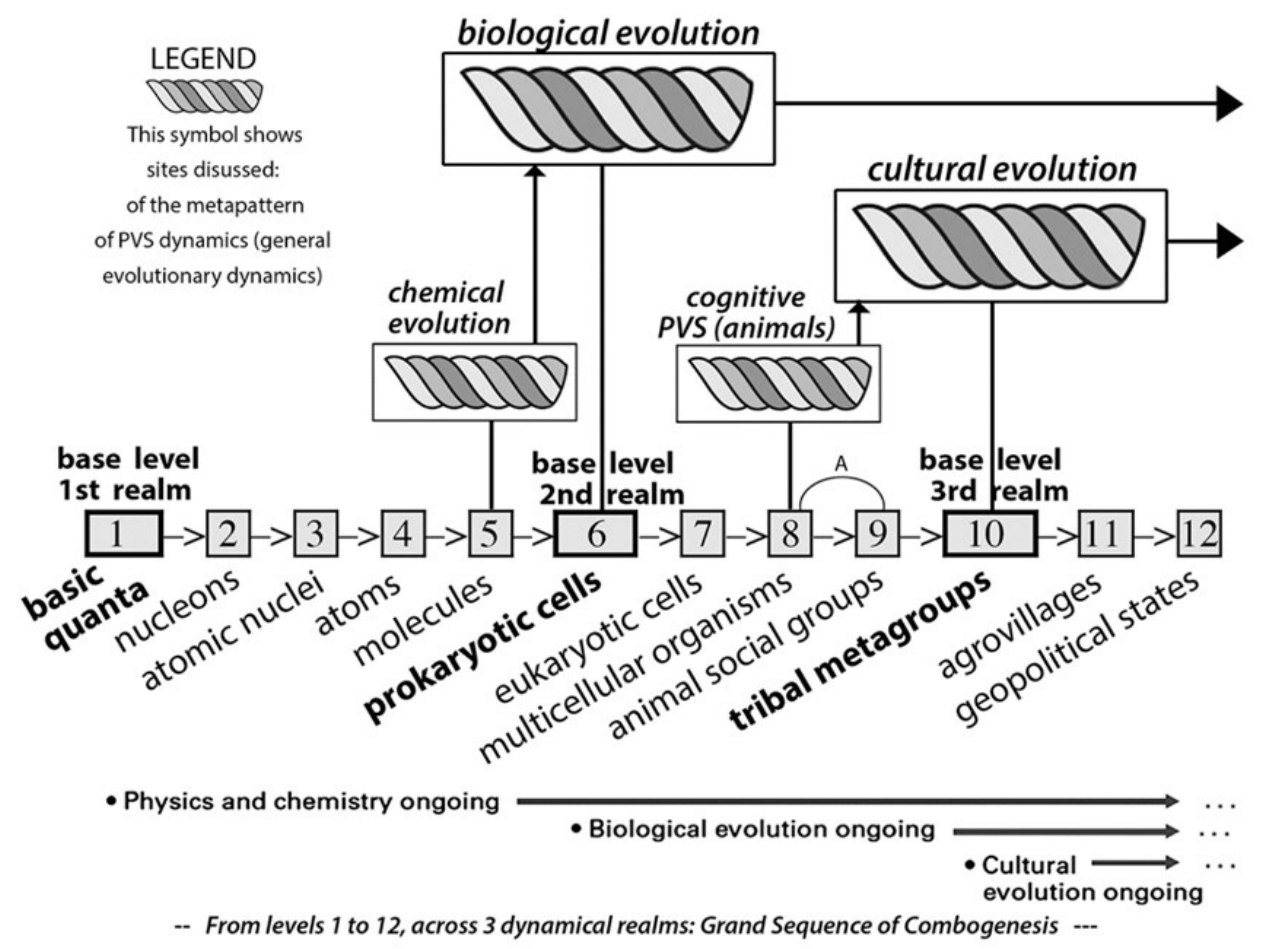

Figure 3. This shows the locations of the instantiations of PVS dynamics discussed in the paper. The two large boxes of the icon (see legend above) show the locations of the respective starts of biological and cultural evolutionary dynamics. The two smaller boxes of the icon show the locations of the evolutionary dynamics of chemical evolution in the realm of physical law and animal cognitive learning in the realm of biological evolution. The letter " $\mathrm{A}$ " is placed to indicate biological evolutionary feedbacks between animal cognitive PVS and animal social groups. The start of cultural evolution took the animal cognitive PVS and advanced it into the human mind's capability for simulation and decision-making, and also included social decision-making, as described in the text, and thus the direct link from animal social groups (with very weak group decision-making, certainly nothing linguistic) and human tribal metagroups, i.e., groups of groups, and on the path of combogenesis from animal groups to human groups of groups. 
Second: the emergence of culture: Within the realm of biological evolution, animals (level 8, see Figure 1) were able to evolve cognitive PVS "learning" dynamics as adaptations that enhanced their lives. Types of animals in more complex social groups (level 9) were able to evolve into self-aware humans with their more sophisticated cognitive PVS systems, living in human tribal metagroups, or groups of groups (level 10), with language and material culture binding the crucially networked local groups within the much larger metagroup of a cultural millieu. Thus, the animal mind with cognitive-PVS became complex human mental PVS and this co-evolved with the special, new social PVS systems for cultural transmission and deliberative group decision-making that came into being. Together, the coupled cognitive and social PVSes generated the overall dual -tier PVS of cultural evolution. See figure 3.

Thus 'advanced' systems in both the realms of physical laws and biological evolution developed a kind of evolutionary dynamics that was still within their realms but then was also able to transition into more radically new forms of evolutionary dynamics. These new dynamics, respectively, and extraordinarily potent at exploring possibility space, started the evolutionary realms of biology and culture.

\section{A Summary of These Parallels as Relevant to Big History}

We can now utilize the metapattern of PVS dynamics to create a grand sequence for big history, with the main plot line following sequential instantiations of the metapattern:

The dynamical realm of physical law began with the fundamental particles of physics, initiating what became a nested climb via serial events of combogenesis toward more complex types of things, until populations of one of those types, namely the molecules, were able to enter into chemical evolutionary dynamics, which then transitioned (how is still quite mysterious) into the origin of life and biological evolutionary dynamics. In that dynamical realm of biological evolution, biological PVS-dynamics continued to produce new patterns, including serial events of combogenesis, until animal cognition was able to obtain a degree of learning complex enough to be called a cognitive PVS dynamics. This continued to evolve in animals within the context of certain animal social groups until, in an important transition of combogenesis, language and material culture could connect groups into groups of groups, or metagroups of humans. In this dynamical realm of cultural evolution, those metagroups contained dual-tier cultural evolutionary dynamics: individual cognitive PVS coupled to a complex social PVS. Subsequent serial events of combogenesis led to the next fundamental levels of agrovillages and geopolitical states, all within the realm of cultural evolution.

It is, therefore, possible both to frame and also further analyze important steps in big history using PVS as a core principle of generalized evolutionary dynamics, both to distinguish and to a large extent define three dynamical realms and also to better understand high-level types of modes of pattern-formation from possibility spaces.

\section{The Number of Realms, Orders, Dimensions}

The view in this paper supports a threefold arc of big history, defined by two major innovations in PVS dynamics: biology and culture. However, others have made cases for more than three main stages. I will discuss two of these alternatives.

First, Lawrence Cahoone has proposed five "orders of nature" (Cahoone 2013). They are the physical, the material, the biological, the mental, and the cultural. I cannot do justice to his thought-provoking, book-length treatment in a few sentences and, therefore, will limit my remarks to the several most relevant.

Cahoone develops an ordering of "increasing complexity," one that distinguishes a "small set [five] of wide strata with properties distinctive enough to be the objects of differing sciences arranged in a hierarchy of dependence and complexity."

Below, I will return to Cahoone's order of the mental ("activities of certain neurologically complex animals species"). For now, let us note that his physical and material orders would together fall under the single, physical column ("Dyn. realm 1") of Table 1, or what I call the dynamical realm of physical law. More detail on these two of 
Cahoone's orders that should be compared to the single stage of Table 1's other scholars is not possible here.

Second, and recently in this journal, Greg Henriques and colleagues (2019) in a highly relevant paper reviewed Henriques' prior work on four "dimensions of existence": Matter, Life, Mind and Culture. Their goal was to compare and contrast the four dimensions of this "Tree of Knowledge System" (ToK) to David Christian's eight thresholds. Their dimension of Matter corresponds to the column of "Dyn. realm 1" of Table 1. Their dimension of Culture corresponds to the column of "Dyn. realm 3" of Table 1. So far, so consistent, but what about their other two dimensions, namely, of Life and Mind?

In the ToK, the dimensions of Life and Mind are closely equivalent to Cahoone's two orders of the biological and the mental. As described, ToK's dimension of Mind began with animals with complex enough brains. Furthermore, the "brain and neural networks are to an animal what DNA and genes are to a cell: a centralized, information relay and storage system." Indeed, following ToK's dimension of Matter, the dimensions of Life, Mind, and Culture are all analyzed to "emerge as a function of different semiotic or information processing systems" (Henriques et al., 2019: quotes from pages 11 and 1, respectively).

Though this emphasis on innovations of information processing as main criteria that defined new dimensions sounds quite different from my proposal for defining new dynamical realms by major innovations in PVS dynamics, the proposals are quite similar in many aspects. Specifically, Henriques and colleagues make specific points that the new dimensions of information processing all have new kinds of feedback loops of "variation, selection, and retention" that produce the various subtypes of complexity within each respective dimension. Thus, after 'my' realm of physical laws (their Matter), Henriques and colleagues basically define subsequent "joint points" (i.e., major transitions) into the emergence of new dimensions by new PVS-dynamical cycles of causation.

Here a key and worthwhile future conversation might involve the placement of animal cognition.
In my proposed system of combogenesis and the grand sequence, the cognitive-PVS of animals (as described above) is not a separate dynamical realm but within the realm of biological evolution. I am going to leave this discussion hanging with that point. I need to contemplate the difference in more detail, hopefully in another paper. Indeed, the five "orders" of Cahoone and the four "dimensions" of ToK are both models worthy of further consideration.

\section{Outstanding Issues, Concluding Remarks}

I would be pleased to see the ideas developed here discussed and debated. I have made a case for three dynamical realms, which closely map to the threefold arcs of other models (Table 1). The key here has been to propose major innovations in the sequential addition of PVS dynamics, first with biology and then with culture, as defining characteristics of new dynamical realms.

I have noted that all models of others in this paper have given some nod to something similar going on in the operations of biology and culture. The metapattern of PVS dynamics is what I have tried to bring forward as an explicit, foundational principle for big history and for the drawing of patterns out from possibility spaces into reality spaces.

Thus, I suggest that to move ahead with the scholarly field of big history, we should consider PVS as a repeating principle-a metapatternthat is powerful and thus momentous. PVS dynamics opens up ways of exploring possibility spaces and gaining actual patterns that can undergo further thresholds or, in my terms, events of combogenesis.

The metapattern of PVS dynamics, thus, would join other proposed principles, such as Goldilocks gradients, energy fluxes, types of complexity, and, potentially, information (if carefully defined for broader applications). Future progress should continue making connections among principles relevant to big history. In addition, my proposal leaves open a number of other avenues of inquiry that seem to me ready for and worthy of further development. I will briefly discuss several. 


\section{What Is This Kind of Logic That Uses Meta- patterns such as PVS Dynamics?}

I am perplexed by this issue. What is the epistemological basis for principles of big history that evoke words and diagrams but not necessarily math? For example, the principle of PVS dynamics cuts across biology and culture. Even more cross-cutting is Christian's metapattern of "pattern itself." Similarly, Spier works with "building blocks, connections, and sequences." Are we dealing with a science (or study) of all pattern? A scholarship-based ontology? A patternology?

Spier clearly sees the issue. He asks, "how would we rate the different aspects [of things we want to connect] and which equations would we use?" He concludes, "for the time being, we have to rely on qualitative, rather than subjective statements of how to assess all the levels of complexity in the known universe" (Spier 2015, 50).

Stuart Kauffman (2019) sees the issue, too. Evolution, even though quantifiable in equations when put in terms of populations of competing degrees of fitness, produces a logic of patterning that goes beyond math. Key is the emergence in biology of "function." One is never (at least not yet) going to mathematize all possible functions that a new biological adaptation is capable of evolving into during the subsequent course of evolution.

Similarly, consider the work of the former president of the International Society for Systems Science, Len Troncale, and the systems theorist George Mobus. They focus on logical concepts such as, respectively, integration-diversification cycles, and an ontology of system properties (see note 3). The point is that there is significant progress using techniques that are not math-based. In the scholarship of big history itself, the Goldilocks gradient is a repeating principle expressed in verbal logic, not requiring math to explain, and perhaps not easily made into math in all cases. I make these remarks and point to just a few others who weigh in on this issue of the type of logic. I personally would like to see more work on these ideas.

\section{Use of the Term "Evolution"}

This is another issue I would like to see debated. Look how many scholars in Table 1 use the term evolution not just for biology and culture, but for their prebiological realm 1. Many even call the entire flow of big history "cosmic evolution" or "universal evolution."

On one hand, I am fine with the use of the term "cosmic evolution." I know (or think I know) what the speaker is referring to: the whole shebang, the cosmic promenade, pageant, or cavalcade from quarks to culture. For example, John Smart (2008) has developed a 'big history' concept of the "evo-devo universe." His concept synthesizes both the processes of evolution and development to refer to cosmic evolution and its repeating patterns across scales. Space does not allow me to discuss this interesting model in more detail here.

On the other hand, I am less than sanguine about dubbing the whole shebang of time as an "evolution." Yes, the general dynamics of both biology and culture are indeed worth terming "evolutionary" (with care, to distinguish things subject to direct evolution from those that are merely affected by the directly evolving things; ecosystems, for example, do not evolve according to my preferred usage). However, the metapattern of PVS dynamics in biology and culture is something quite different from how patterns change in the physical cosmos.

Though many astronomers refer to the 'evolution' of stars and galaxies, their usage of the term is loose, referring to directional change. They are not doing big history, nor stating that stars and galaxies have PVS in the same deep way that is shared by biology and culture. Joseph Voros (2019, 63-64) notes two camps that are using the term "cosmic evolution" (for the nonbiological physical cosmos versus the entire promenade from quarks to culture). His issue is different from the one I am raising, but I commend him for acknowledging variants, or at least preferences, in using the term evolution in the context of big history.

On yet another hand, some have informally pointed out to me there is a kind of variation in atomic nuclei as they are forged in stars, and also 
a kind of selection as certain nuclei stabilize in islands of minimizations of energy potential. Thus, from this viewpoint there can be argued to exist some sort of selection dynamics even in the nonbiological cosmos.

Even so, I will say, that if we use "evolution" for everything from quarks to culture, then we need a distinct term for what is going on in biology and culture that is shared between them and also distinctly different from what is going on with galaxies, stars, and planets. I thus leave this discussion as a hanging issue.

\section{Three Main Contexts for the Things in the Nested Build-up of the Grand Sequence}

In the diagrams presented by Voros (2019) for "evolution" of the physical, biological, and cultural stages, connections between macro and micro scales are prominent for all three of the stages. Similarly, Christian (2018) and Spier (2015) weave back and forth between micro and macro as they discuss changes in their types of complexity.

What I propose here is that we might aim to formalize a relationship between the micro and macro scales at the three dynamical realms. For this, I follow the inspiration provided by Voros, as noted, and also by Cahoone (2013), who borrowed the word "causal thicket" from the philosopher of biology William Wimsatt (1994). Systems of change do so within causal thickets of context initiated with the start of the major "orders."

For example, at the Big Bang a cosmos started. Within that causal thicket we had the internal, nested build-up of levels of combogenesis from fundamental particles to molecules, as well as a myriad of types of meso- and macro-scale things, such as (and not in temporal order) planets, galaxies, stars, nebulae, gases, layers of the Earth, mountains, and oceans. We might say that the changes within the realm of physical laws took place within a largest surrounding context, or causal thicket, of a "cosmosphere."

In my view, then, Earth's biosphere began with the first prokaryotic cells that required inputs of nutrients and exports of wastes (Volk 1998). Living things in the biosphere's interconnected system of atmosphere, ocean, soil, and life do require the sun coming downward through that great "sphere's" upper boundary, and also require the volcanic gases that ascend upward through its lower boundary. But the biosphere is the material container or cauldron for the changes that take place within and across communities, populations, ecosystems, food webs-namely, multiple types of micro-, meso-, and macro-things that require biology for their creation (but not only biology), and which have occurred and altered during the evolution of life for nearly four billion years. We might say that the changes within the dynamical realm of biological evolution took place within a largest context, or causal thicket, of the "biosphere" (Volk, 2009).

For the main context for my proposed third dynamical realm of cultural evolution, we frankly need a word. By the middle or upper Paleolithic (and perhaps earlier), people would have encountered and consciously noted traces of others outside one's specific tribal metagroup. Think of trodden paths, former campsites of fires with areas of debris where took place food preparation or flint knapping and the cave art beheld long after the creators became ancestors. This cultural container is contiguous with the biosphere, but it got specially shaped and folded together as a surrounding matrix of ongoing things of globally connected cultural evolution (albeit incredibly sparse at the start). This matrix continued upward in time into and through the things of agriculture and upward to today's technological systems with their massive impacts to an era of the "anthropocene."

What about a word for this big, planetary culture-affected context, which started with humans, their languages, and their artifacts? Anthroposphere? Culturosphere? Noosphere? Is noosphere too limited to the mental? Some might like "technosphere." To me, that also is too limited for this purpose. Planetary civilome? For the moment, I like civisphere.

In summary, this proposal is that the start of each dynamical realm also started a super-sphere (or super-crucible, super-context, super-causal thicket) within which the myriad things of a given realm were formed and are forming, guided by the dynamics of the respective realms. (Things across the realms do interact with each other, of 
course.) All super-spheres have multiple scales of things within them. I offer this proposal as open to discussion. Refining these ideas might give us an ability to cross-compare patterns at various meso-scales within the dynamical realms.

\section{Using Principles of Big History to Help Us Think about Challenges Faced by Humanity Today}

Much of the impetus for those who are working toward principles of big history comes from the fact that principles can help us clarify, frame, and hopefully propose solutions or new analytical perspectives for global challenges taking place and looming large. I believe that the elucidation and potential application of grand principles is worth our attention.

One can cite almost all those referenced in this paper as examples of scholars connecting big history with the ongoing challenges; space does not allow more detail here. I will suggest that the metapattern of PVS dynamics has a role to play in this endeavor. Specifically, human today are creating a new form of PVS dynamics, in the AI evolutionary systems (deep learning, algorithms that get refined to sell us stuff or surveil us, to improve traffic flows, etc.). Might these new PVS dynamics be one indication that a new level of combogenesis is starting to operate with humanity at the planetary scale? That would be level 13 of my proposed grand sequence. Could these new PVS dynamics indicate a new dynamical realm, which would be a fourth part of the overall big history arc $\left(Q_{2} C\right.$ Epilogue $)$ ?

The new, eternalized PVS of technical and AI systems as decision-making new 'things' is a general concern, as well as possible helpmate for humanity. I personally see these new PVS systems as a definite concern for the future integrity of human nature, a concern that is not being discussed nearly enough in our current traditions and institutions of dual-tier PVS cultural dynamics. These new PVS systems are (so far) external to the human body, and different from traditional technologies, which were mostly under the control of individual cognitive and social PVS of the dual-tier decision-making processes of cultural evolutionary dynamics. Might we be adding another tier of
PVS dynamics to cultural evolution, and, if so, what is that shaping for us as time moves ahead?

\section{Concluding Remarks}

To be interested in all things - the big bang, stars, galaxies, planets, the first cells, animals, trees, human hunter-gatherer bands, the ancient civilizations-is to be interested in general pattern generation. To participate in this scholarly field, one integrates past the boundaries of disciplinary fields and considers how pattern-making operates over the entire quarks-to-culture cavalcade.

According to the analysis here, biological evolutionary dynamics emerged (or developed) from a dynamical realm of physical laws that had, at the level of molecules, an advanced kind of complexity with chemical evolutionary dynamics. Within those biological evolutionary dynamics developed animal cognitive evolutionary dynamics. That adaptation was able to complexify within the context of animal social systems, eventually leading to culture-embedded humans in expandable metagroups with cultural evolutionary dynamics. Thus, a second major type of realmforming evolution dynamics came from the first (culture from biology). The proposal in this paper is that this threefold division-of physical laws, biological evolution, and cultural evolutionmakes sense because we are able to define transitions in pattern-making processes that came from new forms of PVS-dynamics in biology and culture. The metapattern of general evolutionary dynamics manifested at various points of the cosmic cavalcade and thus can both define and tie together major features of the threefold arc of big history.

\section{References}

Bateson, Gregory. 1979. Mind and Nature: A Necessary Unity. New York: Dutton.

Boehm, Christopher. 2012. Moral Origins: The Evolution of Virtue, Altruism, and Shame. New York: Basic.

Boyd, Robert, and Peter J. Richerson. 1985. Culture and the Evolutionary Process. Chicago: University of Chicago Press. 
Buskes, Chris. 2013. "Darwinism Extended: A Survey of How the Idea of Cultural Evolution Evolved." Philosophia 41: 661-691. doi: 10.1007/ s11406-013-9415-8.

Campbell, Donald T. 1960. "Blind Variation and Selective Retentions in Creative Thought as in other Knowledge Processes." Psychological Review 67: 380-400.

Cahoone, Lawrence. 2013. The Orders of Nature. Albany: State University of New York Press.

Chaisson, Eric J. 2014. "The Natural Science Underlying Big History." The Scientific World Journal 2014: Article ID 384912, 41 pages.

Chaisson, Eric J. 2015. "Energy Flows in LowEntropy Complex Systems." Entropy 17: 80078018.

Christian, David. 2011. Maps of Time: An Introduction to Big History. Berkeley: University of California Press. (Pages quoted are from this second edition, paperback.)

Christian, David. 2018. Origin Story: A Big History of Everything. New York: Little, Brown and Company.

Cziko, Gary. 1995. Without Miracles: Universal Selection Theory and the Second Darwinian Revolution. Cambridge, MA: MIT Press.

Dennett, Daniel. 1995. Darwin's Dangerous Idea: Evolution and the Meanings of Life. New York: Simon and Schuster.

Gustafson, Lowell. 2017. "Science, the Deep Past, and the Political." Social Sciences 6: 196-208.

Hayden, Brian. 2012. "Neandertal Social Structure." Oxford Journal of Archeology 31: 1-26.

Henriques, Gregg, Joseph Michalski, Steven Quackenbush, and Waldemar Schmidt. 2019. "The Tree of Knowledge System: A New Map for Big History." Journal of Big History 4 (4): 117.

Hodder, Ian. 2012. Entangled: An Archaeology of the Relationships Between Humans and Things. Chichester, U.K.: Wiley-Blackwell.

Jantsch, E. 1980. The Self-organizing Universe: Scientific and Human Implications of the Emerging Paradigm of Evolution. New York: Pergamon Press.

Kahneman, Daniel. 2011. Thinking, Fast and Slow. New York: Farrar, Straus and Giroux.
Kauffman, Stuart A. 2019. A World Beyond Physics: The Emergence of Life E Evolution. Oxford: Oxford University Press.

Laland, Kevin N. 2017. Darwin's Unfinished Symphony: How Culture Made the Human Mind. Princeton: Princeton University Press.

LeDoux, Joseph. 2019. The Deep History of Ourselves: The Four-Billion-Year Story of How We Got Conscious Brains. New York: Viking.

Luhmann, Niklas. 2012. Theory of Society. Vol. 1. Translated by Rhodes Barrett. Stanford: Stanford University Press.

Pearce, Jack. 2018. Fundamentals for the Anthropocene. Berlin: De Gruyter Open.

Mobus, George E., and Michael C. Kalton. 2015. Principles of Systems Science. New York:

Springer-Verlag.

Plotkin, Henry. 2003. The Imagined World Made Real: Towards a Natural Science of Culture. (Paperback) New Brunswick, N.J.: Rutgers University Press.

Popper, Karl R. 1972. Objective Knowledge: An Evolutionary Approach. Oxford: Clarendon.

Pross, Addy. 2012. What Is Life? How Chemistry Becomes Biology. Oxford: Oxford University Press.

Pross, Addy, and Robert Pascal. 2017. "How and Why Kinetics, Thermodynamics, and Chemistry Induce the Logic of Biological Evolution." Beilstein, J. Organic Chemistry 2017: 665-674.

Renn, Jürgen. 2020. The Evolution of Knowledge: Rethinking Science for the Anthropocene. Princeton: Princeton University Press.

Rolston III, Holmes. 2010. Three Big Bangs: Matter-Energy, Life, Mind. New York: Columbia University Press.

Salk, Jonas. 1983. Anatomy of Reality: Merging of Intuition and Reason. New York: Columbia University Press.

Salk, Jonas. 1985. "The Next Evolutionary Step in the Ascent of Man in the Cosmos." Leonardo 41 (3): 281-286.

Schrödinger, Erwin. 1945. What is Life? The Physical Aspect of the Living. New York: Macmillan.

Smart, John M. (2008) "Evo Devo Universe? A Framework for Speculations on Cosmic Cul- 
ture.” In Dick, Steven J. and Mark Lupisella, eds. Cosmos and Culture. NASA.

Spier, Fred. 2015. Big History and the Future of Humanity. Chichester, UK: Wiley-Blackwell. Originally published 2010. Page numbers in this paper refer to the 2015 second (paperback) edition.

Stewart, John E. 2019. "The Trajectory of Evolution and Its Implications for Humanity." Journal of Big History 3 (3): 141-155.

Szathmáry, Eörs. 2015. "Toward Major Evolutionary Transitions Theory 2.o." Proceedings of the National Academy of Sciences of the United States of America 112, no. 33: 10104-10111. doi: 10.1073/pnas.1421398112.

Tomasello, Michael. 2019. Becoming Human: A Theory of Ontogeny. Cambridge. MA: Belknap. Troncale, Lenard. 1989. Incoming President Plenary, International Society for Systems Science, Portland, Oregon. The current version, as an expanded PowerPoint of that talk is available as "Len. Troncale.USOO.UAA2 I_F Cycles.pdf."

Volk, Tyler. 1995. Metapatterns Across Space, Time, and Mind. New York: Columbia University Press.

Volk, Tyler. 1998/2003. Gaia's Body: Toward a Physiology of the Earth. Paperback ed. Cambridge, MA.: MIT Press, paperback, 2003.

Volk, Tyler. 2009. "How the Biosphere Works." In Crist, E. and B. Rinker, eds. Gaia in Turmoil: Climate Change, Biodepletion, and Earth Ethics in an Age of Crisis. MIT Press, 27-40.

Volk, Tyler. 2017. Quarks to Culture: How We Came to Be. New York: Columbia University Press.

Volk, Tyler, and Bloom, J. W. 2007. "The Use of Metapatterns for Research into Complex Systems of Teaching, Learning, and Schooling, Part I: Metapatterns in Nature and Culture." Complicity: The International Journal of Complexity and Education 4: 25-43. (Available online from the journal or at http:// pages.nyu.edu/ tv1/Volk.htm).

Voros, Joseph. 2019. "Big History in its Cosmic Context." Journal of Big History 3 (3): 57-8o.
Wilson, David Sloan. 2007. Evolution for Everyone: How Darwin's Theory Can Change the Way We Think About Our Lives. New York: Bantam Dell.

Wimsatt, William C. 1994. "The Ontology of Complex Systems: Levels of Organization, Perspectives, and Causal Thickets." Canadian Journal of Philosophy 24, Supl. 1: 207-274.

\section{Endnotes}

${ }^{1}$ I personally regard this as a significant sentence in the BH literature. My own, sympatico viewpoint: "What if we were to truly embrace everything in a study of everything? Then, would the phenomena being studied involve pattern itself? I think so. Everything that can be studied has pattern, from atoms to societies." (Volk, 2017: 9)

${ }^{2}$ I do think a new level 13 of the planetary scale as a combogenesis of nations might be abirthing (Q2C Epilogue). This is similar to the concerns of other big historians in their positing of a new threshold happening right now, and the fact that we can learn from previous "successes" of pattern creation, to seek principles to help formulate this new level.

${ }^{3}$ After Q2C was published, I became aware of intriguing overlaps with concepts developed by systems theorists Len Troncale ("concrescence," Unbroken Sequence of Systems' Origins) and George Mobus ("ontogenesis"). Their models are more general than mine, for they include, for example, stars. Without denying any importance of stars in forming the pathway to human emergence, for the logical purposes I pursued, I limited the "grand sequence" to a nested build-up that progresses strictly from small to large, as I developed a case for what each new level had that could produce to the next. For complementarity in what is happening in the field of systems theory, both these researchers are worth looking into. See Troncale (lentroncale.com, or https:// vimeo.com/363045415) 1989, and Mobus (2015).

${ }^{4} \mathrm{I}$ noted $\left(\mathrm{Q}_{2} \mathrm{C}, 228\right)$ terms used by others for "possibility space," such as "design hyperspace," "adjacent possible," "design space," and "nonmanifest order." Stewart (2019) uses 
"possibility space." Renn (2020) uses "horizon of possibilities."

${ }^{5} \mathrm{~A}$ word about my preferred term, "propagation," rather than the term "inheritance" that many (but not all) use. I can see the advantage to "inheritance," with ideas being transmitted horizontally and vertically, like DNA. I also would insist that we use a term that easily includes material artifacts. This follows archeology's focus on material culture as an integral part of culture. Cars are propagated. (OK, they do not self-propagate, but humans propagate those material patterns.) I do see that inheritance could be stretched to fit. I've wrestled with the issue quite a bit, and obviously it depends on personal comfort in interpretation of the meaning and application of words. In the end, I personally think the word propagation is better because inheritance is more likely to keep one's mind overly (in my opinion) attached to thinking that cultural evolution is mostly about informational pattern transfer among humans. Instead, I would side with Renn (2020), who sees material culture as exerting a degree of regulatory control (by its pattern transfer) on humans, so there is mutual feedback, which also closely follows the concept of "entanglement" between humans and things by Hodder (2012).

${ }^{6}$ We see actions of cultural PVS in dynamics of cars as a system of change over time: designers play with options in their minds for new models (mental, cognitive PVS). The social comes into play as well. Plans are passed up and down the corporate hierarchy. Eventually, new heavy metal realities are produced. They are then chosen and driven by the public. The hopes from the designers and company are for success, but that is not guaranteed. The market adds another layer of the selective (S) sub-process. Successful models can be iterated in greater quantities of near clones, or modified to form lineages, as exponential propagation of both models and lineages of models is possible. Extinctions abound, too. At many scales the various forms of individual cognitive and social PVS are coupled. 